\title{
Communication
}

\section{Copper-Catalyzed Redox Coupling of Nitroarenes with Sodium Sulfinates}

\author{
Saiwen Liu ${ }^{1, *}$, Ru Chen ${ }^{2}$ and Jin Zhang ${ }^{1, *}$ \\ 1 College of Materials and Chemical Engineering, Hunan City University, Yiyang 413000, China \\ 2 Yiyang Agriculture Products Quality Detect Center, Yiyang 413000, China; chenrudaxia@163.com \\ * Correspondence: liusaiwen7@163.com (S.L.); tccdzdc@163.com (J.Z.)
}

Academic Editor: Derek J. McPhee

Received: 9 March 2019; Accepted: 4 April 2019; Published: 10 April 2019

\begin{abstract}
A simple copper-catalyzed redox coupling of sodium sulfinates and nitroarenes is described. In this process, abundant and stable nitroarenes serve as both the nitrogen sources and oxidants, and sodium sulfinates act as both reactants and reductants. A variety of aromatic sulfonamides were obtained in moderate to good yields with broad substrate scope. No external additive is employed for this kind of transformation.
\end{abstract}

Keywords: copper; redox; additive-free

\section{Introduction}

Nitroarenes are a class of the most readily available starting materials in synthetic organic chemistry. The most important property of nitroarenes is able to undergo various reduction-based reactions, especially for the preparation of primary aromatic amines. The traditional processes mainly rely on using a stoichiometric amount of metal/acid or catalytic hydrogenation [1-6]. Direct use of nitroarenes to form new $\mathrm{C}-\mathrm{N}$ bonds is highly attractive, and great effort has been made on the subject during the past decades [7-9]. However, external reducing reagents such as hydrogen and transition-metals are necessary in most cases [10-13]. Recently, there has been significant interest in the transition-metal-catalyzed C-N bond formation from nitroarenes by use of in situ borrowing-hydrogen strategy [14-19]. Among these transformations, two types of reactions can be summarized according to the resultant products: reductive cyclizations and reductive couplings. They provide convenient access to amine, amide and $N$-heterocycles directly from aromatic nitro compounds [20-31].

$\mathrm{N}$-arylsulfonamides are common building blocks among many pharmaceuticals and biologically active molecules [32-35]. Various efforts have been made for the synthesis of sulfonamides [36-39]. The classical methods mainly use arylamines as the nitrogen sources reacting with sulfonyl chlorides or sodium sulfinates [40-45]. Alternatively, $\mathrm{N}$-arylsulfonamides have been successfully synthesized by the transition-metal-catalyzed cross-coupling of sulfonamides with aryl halides, aryl boronic acids, cyclohexanones, alcohols and hydrocarbons [46-57]. Nevertheless, they suffer from some drawbacks of low atom-, step-, and efficiency. In the case of genotoxic and unstable anilines, the undesired impurities may be obtained during the conversion process. Recently, stable and abundant nitroarenes have emerged as a highly attractive nitrogen source for the construction of $N$-arylsulfonamides. A series of Fe-based coupling reaction of sodium arylsulfinates or arylsulfonyl chlorides with nitroarenes to synthesize $\mathrm{N}$-arylsulfonamides have developed. This kind of transformation required stoichiometric amounts of $\mathrm{NaHSO}_{3}$ or Fe dust as the reductant [58-61]. Notably, Deng and co-workers developed a palladium-catalyzed method for the synthesis of $\mathrm{N}$-arylsulfonamides from arylsulfonyl hydrazides and nitroarenes without external reductants $[62,63]$. It would be highly desirable to develop a process using inexpensive and non-toxic metal such as copper for the concise and facile preparation of $\mathrm{N}$-arylsulfonamides from nitroarenes. We hypothesized sodium sulfinate, which generally served 
as reducing regent and was oxidized in the sulfonylation reactions, would be participated in the reductive coupling reactions of nitroarenes. Herein, we present a copper-catalyzed reductive coupling of nitroarenes using sodium sulfinate as the coupling partner and reducing regent, which gives rise to an alternative access to pharmacologically significant $N$-aryl sulfonamides (Scheme 1 ).

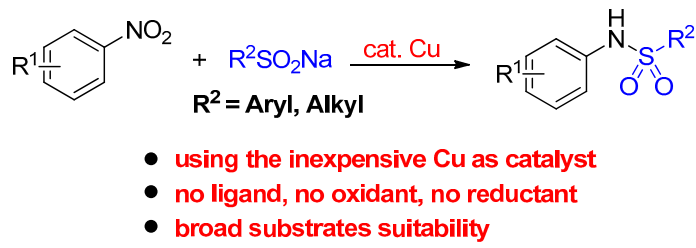

Scheme 1. Copper-catalyzed redox coupling of nitroarenes with sodium sulfinates.

\section{Results}

\subsection{Optimization of Reaction Conditions for Synthesis of 4-Methyl-N-(p-tolyl)benzenesulfonamide 3a}

To begin our study, the reaction of commercially available $p$-methyl nitrobenzene (1a) and sodium 4-methylbenzenesulfinate (2a) was chosen as the model under argon at $120^{\circ} \mathrm{C}$ to optimize the reaction conditions. Three equivalents of sodium sulfinates were used because the substrate served as a reductant. The product $3 a$ was obtained in only $10 \%$ yield without any metal-catalyst (Table 1, entry 1). The use of $\mathrm{FeCl}_{2} \cdot 4 \mathrm{H}_{2} \mathrm{O}$ and $\mathrm{FeSO}_{4} \cdot 7 \mathrm{H}_{2} \mathrm{O}$ afforded $3 \mathrm{a}$ in $20 \%$ and $11 \%$ yields, respectively (entries 2-3). Copper catalyst was found crucial, and various copper salts were investigated for this transformation. Similar results were obtained when employing $\mathrm{CuCO}_{3} \cdot \mathrm{Cu}(\mathrm{OH})_{2}, \mathrm{Cu}(\mathrm{OTf})_{2}, \mathrm{CuCl}_{2}, \mathrm{CuI}$ as the catalyst (entries 4-7). Among the various copper salts examined, $\mathrm{CuCl}$ was the most effective, and its use resulted in the formation of $\mathbf{3 a}$ in $92 \%$ yield (entry 9). $\mathrm{CuBr}$ and $\mathrm{Cu}$ powder also promoted the reaction with slightly lower efficiency (entries 8 and 10). The choice of solvents was important for this reaction. The use of DMF and DMSO reduced the reaction yields to 78\% and 75\%, respectively (entries 11 and 12). Only a trace amount of products were obtained when reactions were carried out in weak polar solvents such as anisole, dioxane, toluene and diglyme (entries 13-16). Unfortunately, a much lower yield was acquired when the reaction was conducted under an atmosphere of air (entry 17).

Table 1. Optimization of the reaction conditions ${ }^{\mathrm{a}}$.

\begin{tabular}{|c|c|c|c|}
\hline Entry & Catalyst & Solvent & Yield $^{\mathrm{b}}(\%)$ \\
\hline 1 & - & NMP & 10 \\
\hline 2 & $\mathrm{FeCl}_{2} \cdot 4 \mathrm{H}_{2} \mathrm{O}$ & NMP & 20 \\
\hline 3 & $\mathrm{FeSO}_{4} \cdot 7 \mathrm{H}_{2} \mathrm{O}$ & NMP & 11 \\
\hline 4 & $\mathrm{Cu}_{2}(\mathrm{OH})_{2} \mathrm{CO}_{3}$ & NMP & 31 \\
\hline 5 & $\mathrm{Cu}(\mathrm{OTf})_{2}$ & NMP & 45 \\
\hline 6 & $\mathrm{CuCl}_{2}$ & NMP & 30 \\
\hline 7 & $\mathrm{CuI}$ & NMP & 46 \\
\hline 8 & $\mathrm{CuBr}$ & NMP & 82 \\
\hline 9 & $\mathrm{CuCl}$ & NMP & 92 \\
\hline 10 & $\mathrm{Cu}$ & NMP & 86 \\
\hline 11 & $\mathrm{CuCl}$ & DMF & 78 \\
\hline 12 & $\mathrm{CuCl}$ & DMSO & 75 \\
\hline 13 & $\mathrm{CuCl}$ & Anisole & 0 \\
\hline 14 & $\mathrm{CuCl}$ & Dioxane & 8 \\
\hline 15 & $\mathrm{CuCl}$ & Toluene & 0 \\
\hline 16 & $\mathrm{CuCl}$ & Diglyme & 15 \\
\hline 17 & $\mathrm{CuCl}$ & NMP & $52^{c}$ \\
\hline
\end{tabular}

\footnotetext{
a Reaction conditions: $1 \mathrm{a}(0.2 \mathrm{mmol}), \mathbf{2 a}(0.6 \mathrm{mmol}), \mathrm{CuCl}(5 \mathrm{~mol} \%), \mathrm{H}_{2} \mathrm{O}$ (2 equiv.), solvent $(0.6 \mathrm{~mL}), 120{ }^{\circ} \mathrm{C}, 40 \mathrm{~h}$, under argon. ${ }^{\mathrm{b}}$ GC yield based on 1a. ${ }^{\mathrm{c}}$ under air.
} 


\subsection{Substrate Scope for the Nitroarenes}

With the optimized conditions in hand, the scope of the reaction with respect to sodium 4-methylbenzenesulfinate (2a) and various nitroarenes was investigated (Table 2). The reaction was found to be general, giving the desired $N$-aryl sulfonamides (3) in reasonable yields. When nitrobenzene was used, the desired product $\mathbf{3 b}$ was achieved in $70 \%$ yield. Common functional substituents such as methoxy and acetyl were compatible with the optimized conditions, and the desired products $3 \mathrm{c}$ and $3 \mathrm{~d}$ were obtained in $68 \%$ and $70 \%$ yields, respectively. Notably, active functional groups such as ester were well tolerated, giving the target products in good yields (3e, 3f). The substituent position on nitroarenes did not show obvious influence on the reaction yields. Moderate yields were obtained when $o$-methylnitrobenzene, $m$-methylnitrobenzene and 2,4-dimethylnitrobenzene were used (3g-3i). Nitroarenes possessing electron-withdrawing group on the phenyl ring also reacted smoothly with $2 \mathbf{a}$ and afforded the desired product in good yield (3j).When naphthalenyl substrate $\mathbf{1 k}$ was used, the desired product $3 \mathbf{k}$ was obtained in $56 \%$ yield. To our delight, hetero-nitroarenes such as 6-nitrobenzothiazole and 5-nitroindole also could react with 2a to give the corresponding sulfonamides in $60 \%$ and $42 \%$ yields, respectively $(31,3 \mathrm{~m})$.

Table 2. Reaction of 2a with various nitroarenes ${ }^{\text {a }}$.

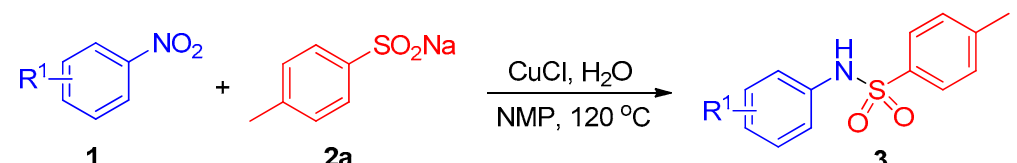

$2 a$<smiles>Cc1ccc(NS(=O)(=O)c2ccc(C)cc2)cc1</smiles>

3a, $77 \%$<smiles>Cc1ccc(S(=O)(=O)Nc2ccccc2)cc1</smiles>

$3 \mathrm{~b}, 70 \%$<smiles>COc1ccc(NS(=O)(=O)c2ccc(C)cc2)cc1</smiles>

$3 c, 68 \%$<smiles>CC(=O)c1ccc(NS(=O)(=O)c2ccc(C)cc2)cc1</smiles><smiles>COC(=O)c1ccc(NS(=O)(=O)c2ccc(C)cc2)cc1</smiles><smiles>CCOC(=O)c1ccc(NS(=O)(=O)c2ccc(C)cc2)cc1</smiles><smiles>Cc1ccc(S(=O)(=O)Nc2ccccc2C)cc1</smiles>

$3 \mathrm{~g}, 55 \%$<smiles>Cc1ccc(S(=O)(=O)Nc2cccc(C)c2)cc1</smiles>

$3 \mathrm{~h}, 68 \%$<smiles>Cc1ccc(S(=O)(=O)Nc2ccc(C)cc2C)cc1</smiles>

$3 i, 64 \%$<smiles>Cc1ccc(S(=O)(=O)Nc2cccc(C#N)c2)cc1</smiles>

$3 \mathrm{j}, \mathbf{7 5 \%}$<smiles>Cc1ccc(S(=O)(=O)Nc2cccc3ccccc23)cc1</smiles>

$3 \mathrm{k}, 56 \%^{\mathrm{b}}$<smiles>Cc1ccc(S(=O)(=O)Nc2ccc3ncsc3c2)cc1</smiles>

$31,60 \%$<smiles>Cc1ccc(S(=O)(=O)Nc2ccc3[nH]ccc3c2)cc1</smiles>

a Conditions: $\mathbf{1}(0.2 \mathrm{mmol}), \mathbf{2 a}(0.6 \mathrm{mmol}), \mathrm{CuCl}(5 \mathrm{~mol} \%), \mathrm{H}_{2} \mathrm{O}$ (2 equiv.), NMP $(0.6 \mathrm{~mL}), 120^{\circ} \mathrm{C}, 40 \mathrm{~h}$, under argon; isolated yield. ${ }^{\mathrm{b}} \mathrm{DMF}$ as solvent.

\subsection{Substrate Scope for the Sodium Sulfinates}

To further examine the scope and the limitations of the reaction, various sodium sulfinates were treated with $p$-methyl nitrobenzene under the standard conditions (Table 3). Sodium benzenesulfinate reacted with 1a smoothly and gave the desired product $3 \mathbf{n}$ in $81 \%$ yield. Other substrates bearing electron-donating group such as methoxy and tert-butyl remained effective and gave the corresponding arylsulfonamides in $72 \%$ and $53 \%$ yields, respectively $(\mathbf{3 o}, 3 \mathbf{p})$. Functional groups halogens were well 
tolerated and afforded the desired products in moderate yields (entries $\mathbf{3 q} \mathbf{q} \mathbf{3} \mathbf{s})$. The reaction showed poor activity when trifluoromethyl group were presented at the para position (3t). Gratifyingly, besides aromatic sodium sulfinates, aliphatic sodium methanesulfinate could also react with 1 a to give the target products in $71 \%$ yield $(3 \mathbf{u})$.

Table 3. Reaction of 1a with various sodium sulfinates ${ }^{\text {a }}$.

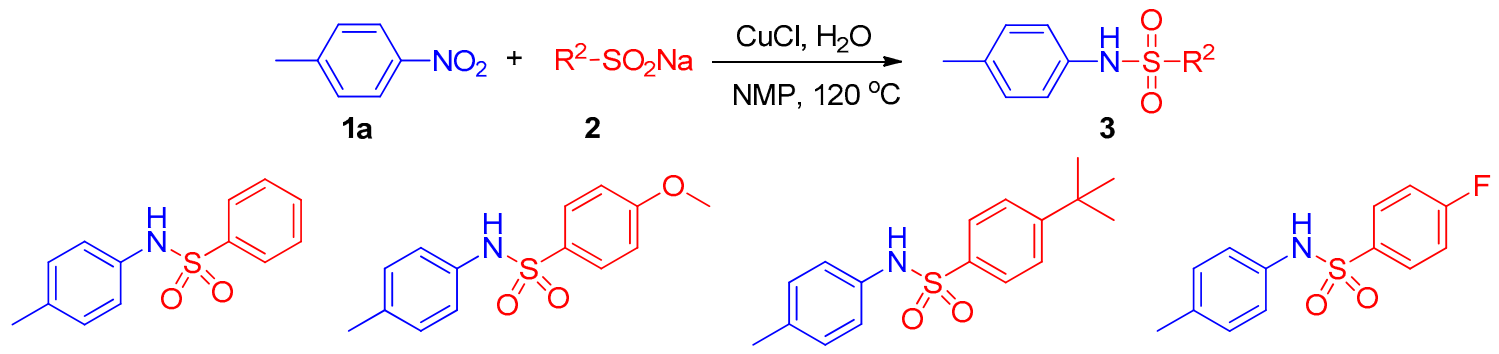

$3 n, 81 \%$

3o, $72 \%$

$3 p, 53 \%$

$3 q, 50 \%$<smiles>Cc1ccc(NS(=O)(=O)c2ccc(Cl)cc2)cc1</smiles>

$3 r, 59 \%$<smiles>Cc1ccc(NS(=O)(=O)c2ccc(Br)cc2)cc1</smiles>

$3 s, 63 \%$<smiles>Cc1ccc(NS(=O)(=O)c2ccc(C(F)(F)F)cc2)cc1</smiles>

$3 t, 40 \%$<smiles>Cc1ccc(NS(C)(=O)=O)cc1</smiles>

$3 u, 71 \%$

a Conditions: 1a $(0.2 \mathrm{mmol}), 2(0.6 \mathrm{mmol}), \mathrm{CuCl}(5 \mathrm{~mol} \%), \mathrm{H}_{2} \mathrm{O}(2$ equiv. $), \mathrm{NMP}(0.6 \mathrm{~mL}), 120^{\circ} \mathrm{C}, 40 \mathrm{~h}$, under argon.

${ }^{\mathrm{b}}$ Isolated yield.

\subsection{Mechanism}

To gather more information about the reaction mechanism, a series of control experiments were set up under different reaction conditions (Scheme 2). When the radical scavenger 1,1-diphenylethylene or 2,2,6,6-tetramethyl-piperidine-1-oxyl (TEMPO) was added to the reaction, the desired product 3a was obtained in 37\% and 7\% GC yields, respectively (Scheme 2a). This indicates that the present reaction process perhaps wasn't a free radical mechanism. Treatment of sodium 4-methylbenzenesulfinate 2a with 4-methyl nitrosobenzene or phenylhydroxylamine under the standard reaction conditions afforded the corresponding product in 32\% and 10\% yield, respectively (Scheme 2b), which implies both the two compounds were not involved as the key intermediate in this coupling reaction. Notably, no product was detected when $p$-toluidine reacted with sodium sulfinate (Scheme 2c). This means that sodium sulfinate could not directly reduce nitrobenzene into aniline in this catalytic system.

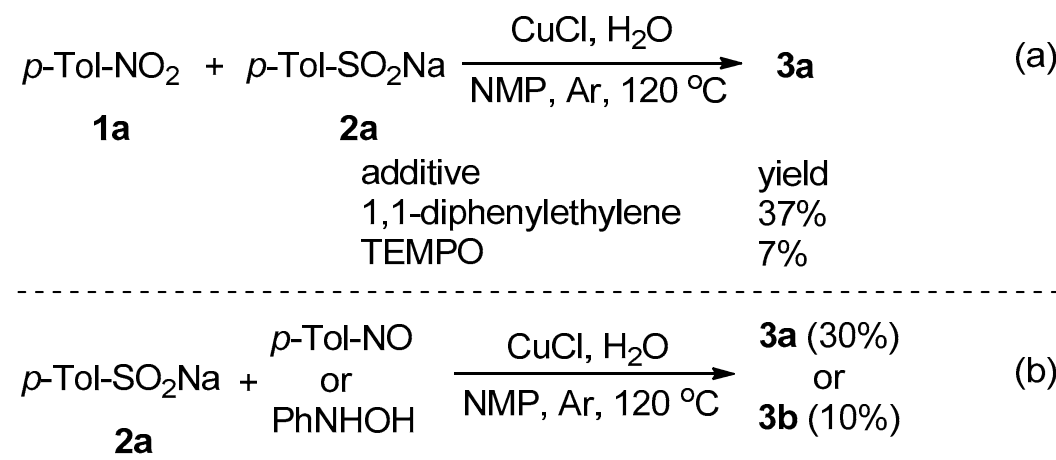

(a)

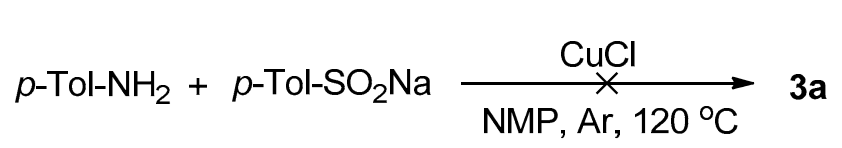

(c)

Scheme 2. Control experiments. 
While the exact mechanism of the reductive coupling of nitroarenes with sodium sulfinate is not clear, as the previously reported nitrobenzene-based reduction [61-63], these mechanistically experimental results encouraged us to propose an inner pathway of the redox process. As shown in Scheme 3, this reaction starts from the coordination between sodium sulfinate 2 and $\mathrm{Cu}(\mathrm{I})$ to form copper(I) sulfinic acid salt A. Then, complexation and nucleophilic addition of the lone electron pair of the sulfur moiety to the nitro group of $\mathbf{1}$ produce a five-membered metallocycle $\mathbf{B}$. Next, reduction of $\mathbf{B}$ by the second molecule of $\mathbf{2}$ generates complex $\mathbf{C}$, followed by the liberation of a sulfonate to afford intermediate D. Subsequently, D continues to be reduced by the third molecule of $\mathbf{2}$ to copper $\mathrm{N}$-arylsulfonamide salt $\mathbf{E}$ along with the sulfonate. At last, protonation of $\mathbf{E}$ affords the desired product and regenerates the $\mathrm{Cu}$ catalyst.

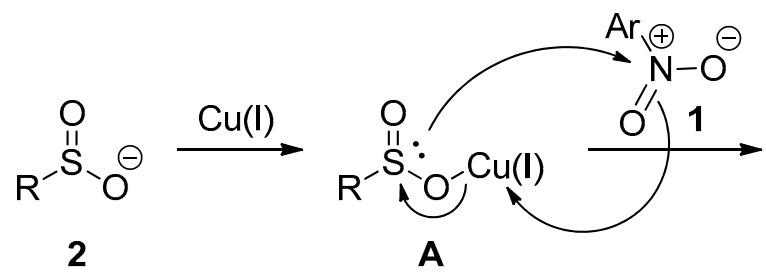

(I)

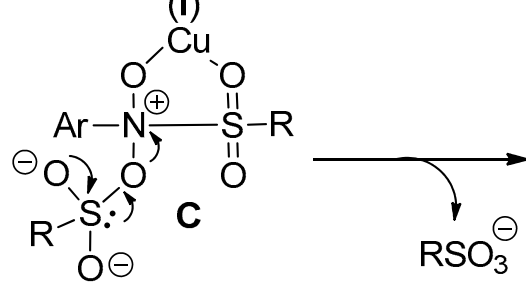<smiles>[R]S1(=O)([O-])O[Ge]ON1[Al]</smiles>

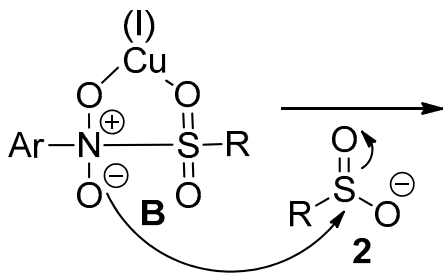<smiles>[R]S(=O)(=O)N([Al])[Al]</smiles>

E<smiles>CC(O)C(C)O</smiles><smiles>[R]S(=O)(=O)N[3H]</smiles><smiles>CC(C)[C@@](C)(C[Se])C(=O)O</smiles>

Scheme 3. Possible reaction mechanism.

\section{Materials and Methods}

\subsection{General Information}

All experiments were carried out under an atmosphere of argon. Flash column chromatography was performed over silica gel 48-75 $\mu \mathrm{m} .{ }^{1} \mathrm{H}-\mathrm{NMR}$ and ${ }^{13} \mathrm{C}-\mathrm{NMR}$ spectra were recorded on Bruker-AV (400 and $100 \mathrm{MHz}$, respectively) instrument (Billerica, MA, USA) internally referenced to $\mathrm{SiMe}_{4}$ or chloroform signals. MS analyses were performed on Agilent 5975 GC-MS instrument (EI) (Santa Clara, CA, USA). The structure of known compounds were further corroborated by comparing their ${ }^{1} \mathrm{H}-\mathrm{NMR},{ }^{13} \mathrm{C}-\mathrm{NMR}$ data and MS data with those of literature. All reagents were used as received from commercial sources without further purification. All nitroarenes and sulfinic acid sodium salts $\mathbf{2 a}, \mathbf{2 b}, \mathbf{2} \mathbf{g} \mathbf{2 h}$, and $\mathbf{2 j}$ employed were reagent grade materials, and others were prepared according to the literature procedures.

\subsection{General Procedure for the Preparation of Sodium Sulfinates}

4-Methoxybenzenesulfinic acid sodium salt (2e) was prepared by heating $2.5 \mathrm{~g}$ of sodium sulfite, $2.06 \mathrm{~g}$ of 4-methoxybenzenesulphonyl chloride, and $1.68 \mathrm{~g}$ of sodium bicarbonate in $9.6 \mathrm{~mL}$ of water at $70-80{ }^{\circ} \mathrm{C}$ for $4 \mathrm{~h}$. After cooling to room temperature, water was removed under vacuum and the residue was extracted by ethanol, recrystallization as a white solid, the yield was $67 \%$ (1.34 g). Similarly, other sodium arenesulfinates were prepared from their corresponding sulfonyl chlorides. 


\subsection{General Procedure for the Synthesis of N-Arylsulfonamides}

A pressure tube $(10 \mathrm{~mL})$ was charged with $\mathrm{CuCl}(1.0 \mathrm{mg}, 0.01 \mathrm{mmol}), p$-toluenesulfinic acid sodium salt (2a, $107.2 \mathrm{mg}, 0.6 \mathrm{mmol}), p$-methyl nitrobenzene $(\mathbf{1 a}, 27.4 \mathrm{mg}, 0.2 \mathrm{mmol})$ and purged with argon three times. NMP $(0.6 \mathrm{~mL})$ and $\mathrm{H}_{2} \mathrm{O}(7.2 \mu \mathrm{L})$ were added by syringe. The resulting solution was stirred at $120^{\circ} \mathrm{C}$ for $40 \mathrm{~h}$. After cooling to room temperature, the crude product mixture was diluted with ethylacetate $(15 \mathrm{~mL})$ and washed with a saturated solution of $\mathrm{NaCl}(3 \times 15 \mathrm{~mL})$ and the organic layer was dried over $\mathrm{MgSO}_{4}$ and concentrated under reduced pressure. The resulting residue was purified by column chromatography (silica gel, petroleum ether/ethyl acetate $=6: 1$ ) to give $3 \mathbf{a}$ as white solid; yield: $40.3 \mathrm{mg}$ (77\%) (NMR spectra for all compounds shown in Supplementary Materials).

\subsection{Product Characterization}

4-Methyl-N-p-tolylbenzenesulfonamide (3a) [58]: White solid, 77\% yield (40.3 mg), ${ }^{1} \mathrm{H}-\mathrm{NMR}(400 \mathrm{MHz}$, $\left.\left.\mathrm{CDCl}_{3}, \mathrm{ppm}\right) \delta 7.64(\mathrm{~d}, J=8.4 \mathrm{~Hz}, 2 \mathrm{H}), 7.24 \mathrm{n}, 2 \mathrm{H}\right), 7.05(\mathrm{~d}, J=8.0 \mathrm{~Hz}, 2 \mathrm{H}), 6.96(\mathrm{~d}, J=8.0 \mathrm{~Hz}, 2 \mathrm{H})$, $6.51(\mathrm{~s}, 1 \mathrm{H}), 2.40(\mathrm{~s}, 3 \mathrm{H}), 2.29(\mathrm{~s}, 3 \mathrm{H}) ;{ }^{13} \mathrm{C}-\mathrm{NMR}\left(100 \mathrm{MHz}, \mathrm{CDCl}_{3}, \mathrm{ppm}\right) \delta 143.7,136.2,135.3,133.9$, 129.8, 129.6, 127.3, 122.2, 21.5, 20.8; MS (EI) $m / z$ (\%) 261, 155, 106 (100), 91, 77, 65.

4-Methyl-N-phenyl-benzenesulfonamide (3b) [58]: White solid, 70\% yield (34.6 mg), ${ }^{1} \mathrm{H}-\mathrm{NMR}(400 \mathrm{MHz}$, $\left.\mathrm{CDCl}_{3}, \mathrm{ppm}\right) \delta 7.64(\mathrm{~d}, J=7.6 \mathrm{~Hz}, 2 \mathrm{H}), 7.26-7.22(\mathrm{~m}, 4 \mathrm{H}), 7.11(\mathrm{t}, J=7.2 \mathrm{~Hz}, 1 \mathrm{H}), 7.05(\mathrm{~d}, J=7.4 \mathrm{~Hz}$, 2H), 6.55 (s, 1H), 2.37 (s, 3H); ${ }^{13} \mathrm{C}-\mathrm{NMR}\left(100 \mathrm{MHz}, \mathrm{CDCl}_{3}, \mathrm{ppm}\right) \delta 143.9,136.7,136.2,129.7,129.3,127.3$, 125.2, 121.5, 21.5; MS (EI) $m / z$ (\%) 247, 182, 168, 155, 91 (100), 65.

4-Methyl-N-(4-methoxyphenyl)-benzenesulfonamide (3c) [58]: White solid, 68\% yield (37.7 mg), ${ }^{1} \mathrm{H}-\mathrm{NMR}$ $\left(400 \mathrm{MHz}, \mathrm{CDCl}_{3}, \mathrm{ppm}\right) \delta 7.58(\mathrm{~d}, J=7.8 \mathrm{~Hz}, 2 \mathrm{H}), 7.24(\mathrm{~d}, J=7.5 \mathrm{~Hz}, 2 \mathrm{H}), 6.98(\mathrm{~d}, J=8.6 \mathrm{~Hz}, 2 \mathrm{H})$, $6.78(\mathrm{~d}, J=8.2 \mathrm{~Hz}, 2 \mathrm{H}), 6.20(\mathrm{~s}, 1 \mathrm{H}), 3.78(\mathrm{~s}, 3 \mathrm{H}), 2.41(\mathrm{~s}, 3 \mathrm{H}) ;{ }^{13} \mathrm{C}-\mathrm{NMR}\left(100 \mathrm{MHz}, \mathrm{CDCl}_{3}, \mathrm{ppm}\right) \delta 157.9$, 143.7, 136.1, 129.6, 129.1, 127.4, 125.3, 114.5, 55.4, 21.5; MS (EI) $m / z$ (\%) 277, 122 (100), 95, 65.

4-Methyl-N-p-acetylphenyl-benzenesulfonamide (3d) [55]: Off-white solid, 70\% yield (44.0 mg), ${ }^{1} \mathrm{H}-\mathrm{NMR}$ $\left(400 \mathrm{MHz}, \mathrm{CDCl}_{3}, \mathrm{ppm}\right) \delta 7.85(\mathrm{~d}, J=8.0 \mathrm{~Hz}, 2 \mathrm{H}), 7.32(\mathrm{~d}, J=7.4 \mathrm{~Hz}, 2 \mathrm{H}), 7.26(\mathrm{~s}, 2 \mathrm{H}), 7.14(\mathrm{~d}, J=7.7 \mathrm{~Hz}$, 2H), 6.88 (s, 1H), 2.53 (s, 3H), 2.38 (s, 3H); ${ }^{13} \mathrm{C}-\mathrm{NMR}\left(100 \mathrm{MHz}, \mathrm{CDCl}_{3}, \mathrm{ppm}\right) \delta 196.6,144.4,141.0,135.9$, 133.4, 129.9, 129.8, 127.2, 119.1, 26.3, 21.5; MS (EI) $m / z$ (\%) 289, 274, 155, 106, 91 (100), 77, 65.

Methyl-4-(4-methylphenylsulfonamido) benzoate (3e) [58]: Off-white solid, 76\% yield (46.4 mg), ${ }^{1} \mathrm{H}-\mathrm{NMR}$ $\left(400 \mathrm{MHz}, \mathrm{CDCl}_{3}, \mathrm{ppm}\right) \delta 7.94(\mathrm{~d}, J=7.8 \mathrm{~Hz}, 2 \mathrm{H}), 7.72(\mathrm{~d}, J=7.6 \mathrm{~Hz}, 2 \mathrm{H}), 7.28(\mathrm{~s}, 2 \mathrm{H}), 7.13(\mathrm{~d}, J=7.8 \mathrm{~Hz}$, 2H), $6.77(\mathrm{~s}, 1 \mathrm{H}), 3.89(\mathrm{~s}, 3 \mathrm{H}), 2.40(\mathrm{~s}, 3 \mathrm{H}) ;{ }^{13} \mathrm{C}-\mathrm{NMR}\left(100 \mathrm{MHz}, \mathrm{CDCl}_{3}, \mathrm{ppm}\right) \delta 166.3,144.4,140.9,132.6$, 131.1, 129.8, 127.3, 126.4, 119.2, 52.1, 21.5; MS (EI) $m / z$ (\%) 305, 155, 122, 91 (100), 65.

Ethyl-4-(4-methylphenylsulfonamido) benzoate (3f) [55]: Off-white solid, 78\% yield (49.8 mg), ${ }^{1} \mathrm{H}-\mathrm{NMR}$ $\left(400 \mathrm{MHz}, \mathrm{CDCl}_{3}, \mathrm{ppm}\right) \delta 7.94(\mathrm{~s}, 2 \mathrm{H}), 7.72(\mathrm{~s}, 2 \mathrm{H}), 7.28(\mathrm{~s}, 2 \mathrm{H}), 7.13(\mathrm{~s}, 2 \mathrm{H}), 6.79(\mathrm{~s}, 1 \mathrm{H}), 4.35(\mathrm{~s}, 2 \mathrm{H})$, $2.40(\mathrm{~s}, 3 \mathrm{H}), 1.38(\mathrm{~s}, 3 \mathrm{H}) ;{ }^{13} \mathrm{C}-\mathrm{NMR}\left(100 \mathrm{MHz}, \mathrm{CDCl}_{3}, \mathrm{ppm}\right) \delta 165.9,144.4,140.8,135.9,131.1,129.8$, 127.3, 126.8, 119.2, 61.0, 21.5, 14.3; MS (EI) $m / z$ (\%) 319, 274, 155, 119, 108, 91 (100), 65.

4-Methyl-N-o-tolyl-benzenesulfonamide (3g) [58]: White solid, 55\% yield (28.7 mg), ${ }^{1} \mathrm{H}-\mathrm{NMR}(400 \mathrm{MHz}$, $\left.\mathrm{CDCl}_{3}, \mathrm{ppm}\right) \delta 7.60(\mathrm{~d}, J=7.7 \mathrm{~Hz}, 2 \mathrm{H}), 7.31(\mathrm{~d}, J=7.8 \mathrm{~Hz}, 1 \mathrm{H}), 7.21(\mathrm{~d}, J=7.6 \mathrm{~Hz}, 2 \mathrm{H}), 7.14(\mathrm{~s}, 1 \mathrm{H})$, 7.08 (s, 2H), 6.23 (s, 1H), 2.39 (s, 3H), 1.99 (s, 3H); ${ }^{13} \mathrm{C}-\mathrm{NMR}\left(100 \mathrm{MHz}, \mathrm{CDCl}_{3}, \mathrm{ppm}\right) \delta$ 143.8, 136.9, 134.6, 131.5, 130.8, 129.6, 127.6, 126.9, 126.2, 124.4, 21.5, 17.6; MS (EI) $m / z$ (\%) 261, 155, 106 (100), 91, 77,65 .

4-Methyl-N-m-tolyl-benzenesulfonamide (3h) [58]: White solid, 68\% yield (35.5 mg) ${ }^{1} \mathrm{H}-\mathrm{NMR}(400 \mathrm{MHz}$, $\left.\mathrm{CDCl}_{3}, \mathrm{ppm}\right) \delta 7.64(\mathrm{~d}, J=7.8 \mathrm{~Hz}, 2 \mathrm{H}), 7.22(\mathrm{~d}, J=7.6 \mathrm{~Hz}, 2 \mathrm{H}), 7.10(\mathrm{t}, J=7.5 \mathrm{~Hz}, 1 \mathrm{H}), 6.92(\mathrm{~d}, J=7.3 \mathrm{~Hz}$, 1H), $6.88(\mathrm{~s}, 1 \mathrm{H}), 6.83(\mathrm{~d}, J=7.5 \mathrm{~Hz}, 1 \mathrm{H}), 6.42(\mathrm{~s}, 1 \mathrm{H}), 2.38(\mathrm{~s}, 3 \mathrm{H}), 2.27(\mathrm{~s}, 3 \mathrm{H}) ;{ }^{13} \mathrm{C}-\mathrm{NMR}(100 \mathrm{MHz}$, $\left.\mathrm{CDCl}_{3}, \mathrm{ppm}\right) \delta 143.8,139.3,136.7,136.3,129.6,129.1,127.3,125.9,122.0,118.3,21.5,21.3$; MS (EI) $m / z$ (\%) 261, 196, 182, 155, 106 (100), 91, 77, 65. 
4-Methyl-N-(2,4-dimethylphenyl)-benzenesulfonamide (3i) [64]: White solid, 64\% yield (35.2 mg), ${ }^{1} \mathrm{H}-\mathrm{NMR}$ $\left(400 \mathrm{MHz}, \mathrm{CDCl}_{3}, \mathrm{ppm}\right) \delta 7.61(\mathrm{~d}, J=7.6 \mathrm{~Hz}, 2 \mathrm{H}), 7.24(\mathrm{~d}, J=7.5 \mathrm{~Hz}, 2 \mathrm{H}), 7.15(\mathrm{~d}, J=7.8 \mathrm{~Hz}, 1 \mathrm{H}), 6.95(\mathrm{~d}$, $J=7.9 \mathrm{~Hz}, 1 \mathrm{H}), 6.92(\mathrm{~s}, 1 \mathrm{H}), 6.18(\mathrm{~s}, 1 \mathrm{H}), 2.41(\mathrm{~s}, 3 \mathrm{H}), 2.28(\mathrm{~s}, 3 \mathrm{H}), 1.96(\mathrm{~s}, 3 \mathrm{H}) ;{ }^{13} \mathrm{C}-\mathrm{NMR}(100 \mathrm{MHz}$, $\left.\mathrm{CDCl}_{3}, \mathrm{ppm}\right) \delta 143.6,137.0,136.2,132.3,131.8,131.5,129.6,127.4,127.2,125.3,21.5,20.9,17.6$; MS (EI) $m / z(\%) 275,120(100), 91,77$.

4-Methyl-N-m-cyanophenyl-benzenesulfonamide (3j) [62]: White solid, $75 \%$ yield (40.8 $\mathrm{mg}),{ }^{1} \mathrm{H}-\mathrm{NMR}$ $\left(400 \mathrm{MHz}, \mathrm{CDCl}_{3}, \mathrm{ppm}\right) \delta 7.69(\mathrm{~d}, J=6.6 \mathrm{~Hz}, 2 \mathrm{H}), 7.38-7.35(\mathrm{~m}, 3 \mathrm{H}), 7.29-7.27(\mathrm{~m}, 2 \mathrm{H}), 7.06(\mathrm{~s}, 1 \mathrm{H})$, $2.41(\mathrm{~s}, 3 \mathrm{H}) ;{ }^{13} \mathrm{C}-\mathrm{NMR}\left(100 \mathrm{MHz}, \mathrm{CDCl}_{3}, \mathrm{ppm}\right) \delta 144.7,137.8,135.6,130.3,130.0,128.5,127.2,125.1$, 123.6, 118.0, 113.4, 21.6; MS (EI) $m / z$ (\%) 272, 155, 91 (100), 65.

4-Methyl-N-(naphthalene-1-yl)benzenesulfonamide (3k) [62]: White solid, 56\% yield (33.3 mg), ${ }^{1} \mathrm{H}-\mathrm{NMR}$ $\left(400 \mathrm{MHz}, \mathrm{CDCl}_{3}, \mathrm{ppm}\right) \delta 7.81(\mathrm{~d}, J=5.4 \mathrm{~Hz}, 2 \mathrm{H}), 7.72(\mathrm{~s}, 1 \mathrm{H}), 7.62(\mathrm{~d}, J=6.4 \mathrm{~Hz}, 2 \mathrm{H}), 7.45(\mathrm{~s}, 2 \mathrm{H})$, $7.37(\mathrm{~s}, 2 \mathrm{H}), 7.16(\mathrm{~d}, J=6.2 \mathrm{~Hz}, 2 \mathrm{H}), 6.79(\mathrm{~s}, 1 \mathrm{H}), 2.34(\mathrm{~s}, 3 \mathrm{H}) ;{ }^{13} \mathrm{C}-\mathrm{NMR}\left(100 \mathrm{MHz}, \mathrm{CDCl}_{3}, \mathrm{ppm}\right) \delta 143.8$, 136.5, 134.3, 131.5, 129.6, 128.9, 128.4, 127.4, 127.2, 126.6, 126.3, 125.4, 122.7, 121.5, 21.5; MS (EI) $m / z$ (\%) 297, $142(100), 115,91$.

$\mathrm{N}$-(Benzo[d]thiazol-6-yl)-4-methylbenzenesulfonamide (31) [65]: Off-white solid, 60\% yield (36.5 mg), ${ }^{1} \mathrm{H}-\mathrm{NMR}\left(400 \mathrm{MHz}, \mathrm{CDCl}_{3}, \mathrm{ppm}\right) \delta 8.96(\mathrm{~s}, 1 \mathrm{H}), 7.98(\mathrm{~d}, J=8.4 \mathrm{~Hz}, 1 \mathrm{H}), 7.84(\mathrm{~s}, 1 \mathrm{H}), 7.67(\mathrm{~d}, J=7.3 \mathrm{~Hz}$, $2 \mathrm{H}), 7.24(\mathrm{~d}, J=7.2 \mathrm{~Hz}, 2 \mathrm{H}), 7.15(\mathrm{~d}, J=8.4 \mathrm{~Hz}, 1 \mathrm{H}), 7.03(\mathrm{~s}, 1 \mathrm{H}), 2.39(\mathrm{~s}, 3 \mathrm{H}) ;{ }^{13} \mathrm{C}-\mathrm{NMR}(100 \mathrm{MHz}$, $\left.\mathrm{CDCl}_{3}, \mathrm{ppm}\right) \delta 154.0,150.8,144.1,136.0,134.8,134.6,129.8,127.3,124.0,121.0,114.6,21.5 ; \mathrm{MS}$ (EI) $\mathrm{m} / \mathrm{z}$ (\%) 304,149 (100), 122, 105, 91, 65.

$\mathrm{N}$-(1H-Indol-5-yl)-4-methylbenzenesulfonamide (3m): White solid, $42 \%$ yield $(24.0 \mathrm{mg}),{ }^{1} \mathrm{H}-\mathrm{NMR}$ $\left(400 \mathrm{MHz}, \mathrm{CDCl}_{3}, \mathrm{ppm}\right) \delta 8.18(\mathrm{~s}, 1 \mathrm{H}), 7.59(\mathrm{~d}, J=7.3 \mathrm{~Hz}, 2 \mathrm{H}), 7.34(\mathrm{~s}, 2 \mathrm{H}), 7.24(\mathrm{~s}, 1 \mathrm{H}), 7.20(\mathrm{~d}$, $J=7.1 \mathrm{~Hz}, 2 \mathrm{H}), 6.90(\mathrm{~d}, J=8.3 \mathrm{~Hz}, 1 \mathrm{H}), 6.49(\mathrm{~s}, 1 \mathrm{H}), 6.31(\mathrm{~s}, 1 \mathrm{H}), 2.38(\mathrm{~s}, 3 \mathrm{H}) ;{ }^{13} \mathrm{C}-\mathrm{NMR}(100 \mathrm{MHz}$, $\left.\mathrm{CDCl}_{3}, \mathrm{ppm}\right) \delta 143.4,136.4,134.3,129.4,128.5,127.4,126.3,125.3,119.5,116.7,111.4,103.0,21.5 ; \mathrm{MS}$ (EI) $m / z(\%)$ 286, 131 (100), 104, 91, 77; HRMS calcd. for $\mathrm{C}_{15} \mathrm{H}_{15} \mathrm{~N}_{2} \mathrm{O}_{2} \mathrm{~S}[\mathrm{M}+\mathrm{H}]^{+}$287.0784, found 287.0779.

$\mathrm{N}$-(4-Methylphenyl)-benzenesulfonamide (3n) [58]: White solid, 81\% yield $(40.0 \mathrm{mg}),{ }^{1} \mathrm{H}-\mathrm{NMR}(400 \mathrm{MHz}$, $\left.\mathrm{CDCl}_{3}, \mathrm{ppm}\right) \delta 7.74(\mathrm{~d}, J=7.6 \mathrm{~Hz}, 2 \mathrm{H}), 7.54(\mathrm{t}, J=7.2 \mathrm{~Hz}, 1 \mathrm{H}), 7.44(\mathrm{t}, J=7.5 \mathrm{~Hz}, 2 \mathrm{H}), 7.05(\mathrm{~d}, J=7.9 \mathrm{~Hz}$, 2H), $6.94(\mathrm{~d}, J=7.9 \mathrm{~Hz}, 2 \mathrm{H}), 6.39(\mathrm{~s}, 1 \mathrm{H}), 2.28(\mathrm{~s}, 3 \mathrm{H}) ;{ }^{13} \mathrm{C}-\mathrm{NMR}\left(100 \mathrm{MHz}, \mathrm{CDCl}_{3}, \mathrm{ppm}\right) \delta 139.2,135.5$, 133.7, 132.9, 129.9, 129.0, 127.3, 122.5, 20.8; MS (EI) $m / z$ (\%) 247, 106 (100), 77, 51.

4-Methoxy-N-p-tolylbenzenesulfonamide (3o) [55]: White solid, $72 \%$ yield (39.9 mg), ${ }^{1} \mathrm{H}-\mathrm{NMR}(400 \mathrm{MHz}$, $\left.\mathrm{CDCl}_{3}, \mathrm{ppm}\right) \delta 7.69(\mathrm{~d}, J=8.5 \mathrm{~Hz}, 2 \mathrm{H}), 7.05(\mathrm{~d}, J=7.6 \mathrm{~Hz}, 2 \mathrm{H}), 6.96(\mathrm{~d}, J=7.6 \mathrm{~Hz}, 2 \mathrm{H}), 6.90(\mathrm{~d}, J=8.4 \mathrm{~Hz}$, 2H), 6.54 (s, 1H), 3.85 (s, 3H), 2.29 (s, 3H); ${ }^{13} \mathrm{C}-\mathrm{NMR}\left(100 \mathrm{MHz}, \mathrm{CDCl}_{3}, \mathrm{ppm}\right) \delta 163.1,135.3,133.9,130.8$, 129.8, 129.5, 122.3, 114.2, 55.6, 20.9; MS (EI) $m / z$ (\%) 277, 171, 106 (100), 77.

4-Tert-butyl-N-p-tolylbenzenesulfonamide (3p) [66]: White solid, yield: $32.1 \mathrm{mg}(53 \%),{ }^{1} \mathrm{H}-\mathrm{NMR}(400 \mathrm{MHz}$, $\left.\mathrm{CDCl}_{3}, \mathrm{ppm}\right) \delta 7.68(\mathrm{~d}, J=7.5 \mathrm{~Hz}, 2 \mathrm{H}), 7.45(\mathrm{~d}, J=7.4 \mathrm{~Hz}, 2 \mathrm{H}), 7.07(\mathrm{~d}, J=7.0 \mathrm{~Hz}, 2 \mathrm{H}), 6.98(\mathrm{~d}, J=6.8 \mathrm{~Hz}$, 2H), 6.39 (s, 1H), 2.30 (s, 3H), 1.32 (s, 9H); ${ }^{13} \mathrm{C}-\mathrm{NMR}\left(100 \mathrm{MHz}, \mathrm{CDCl}_{3}, \mathrm{ppm}\right) \delta 156.7,136.3,135.1,134.0$, 129.8, 127.1, 126.0, 122.0, 35.1, 31.1, 20.8; MS (EI) $m / z$ (\%) 303, 182, 133, 106 (100), 77.

4-Fluoro-N-p-tolylbenzenesulfonamide (3q) [67]: White solid, 50\% yield (26.5 mg), ${ }^{1} \mathrm{H}-\mathrm{NMR}(400 \mathrm{MHz}$, $\left.\mathrm{CDCl}_{3}, \mathrm{ppm}\right) \delta 7.75(\mathrm{t}, J=5.9 \mathrm{~Hz}, 2 \mathrm{H}), 7.12(\mathrm{t}, J=8.5 \mathrm{~Hz}, 2 \mathrm{H}), 7.08(\mathrm{~d}, J=7.9 \mathrm{~Hz}, 2 \mathrm{H}), 6.95(\mathrm{~d}, J=7.8 \mathrm{~Hz}$, 2H), $6.36(\mathrm{~s}, 1 \mathrm{H}), 2.31(\mathrm{~s}, 3 \mathrm{H}),{ }^{13} \mathrm{C}-\mathrm{NMR}\left(100 \mathrm{MHz}, \mathrm{CDCl}_{3}, \mathrm{ppm}\right) \delta 165.2(\mathrm{~d}, J=253 \mathrm{~Hz}), 135.9,135.1(\mathrm{~d}$, $J=3.0 \mathrm{~Hz}), 133.4,130.1(\mathrm{~d}, J=9.4 \mathrm{~Hz}), 130.0,122.7,116.2(\mathrm{~d}, J=22.5 \mathrm{~Hz}), 20.8 ;$ MS (EI) $m / z(\%) 265$, 106 (100), 95, 77.

4-Chloro-N-p-tolylbenzenesulfonamide (3r) [59]: Off-white solid, 59\% yield (33.3 mg), ${ }^{1} \mathrm{H}-\mathrm{NMR}(400 \mathrm{MHz}$, $\left.\mathrm{CDCl}_{3}, \mathrm{ppm}\right) \delta 7.67(\mathrm{~d}, J=7.6 \mathrm{~Hz}, 2 \mathrm{H}), 7.42(\mathrm{~d}, J=7.5 \mathrm{~Hz}, 2 \mathrm{H}), 7.08(\mathrm{~d}, J=6.7 \mathrm{~Hz}, 2 \mathrm{H}), 6.95(\mathrm{~d}, J=6.7 \mathrm{~Hz}$, 2H), $6.45(\mathrm{~s}, 1 \mathrm{H}), 2.31(\mathrm{~s}, 3 \mathrm{H}) ;{ }^{13} \mathrm{C}-\mathrm{NMR}\left(100 \mathrm{MHz}, \mathrm{CDCl}_{3}, \mathrm{ppm}\right) \delta 139.5,139.4,136.2,133.2,130.0,129.3$, 128.7, 122.9, 20.9; MS (EI) $m / z$ (\%) 281, 106 (100), 77. 
4-Bromo-N-p-tolylbenzenesulfonamide (3s) [68]: Off-white solid, 63\% yield (41.0 mg), ${ }^{1} \mathrm{H}-\mathrm{NMR}(400 \mathrm{MHz}$, $\left.\mathrm{CDCl}_{3}, \mathrm{ppm}\right) \delta 7.59(\mathrm{~s}, 4 \mathrm{H}), 7.08(\mathrm{~d}, J=7.3 \mathrm{~Hz}, 2 \mathrm{H}), 6.95(\mathrm{~d}, J=7.0 \mathrm{~Hz}, 2 \mathrm{H}), 6.37(\mathrm{~s}, 1 \mathrm{H}), 2.31(\mathrm{~s}, 3 \mathrm{H})$; ${ }^{13} \mathrm{C}-\mathrm{NMR}\left(100 \mathrm{MHz}, \mathrm{CDCl}_{3}, \mathrm{ppm}\right) \delta 138.2,136.1,133.2,132.3,130.1,128.8,128.0,122.9,20.9$; MS (EI) $m / z(\%) 327,106(100), 77$.

4-Trifluoromethyl-N-p-tolylbenzenesulfonamide (3t) [55]: White solid, yield: $25.3 \mathrm{mg}$ (40\%), ${ }^{1} \mathrm{H}-\mathrm{NMR}$ $\left(400 \mathrm{MHz}, \mathrm{CDCl}_{3}, \mathrm{ppm}\right) \delta 7.87(\mathrm{~d}, J=7.1 \mathrm{~Hz}, 2 \mathrm{H}), 7.72(\mathrm{~d}, J=7.1 \mathrm{~Hz}, 2 \mathrm{H}), 7.09(\mathrm{~d}, J=6.8 \mathrm{~Hz}, 2 \mathrm{H})$, $6.97(\mathrm{~d}, J=6.7 \mathrm{~Hz}, 2 \mathrm{H}), 6.63(\mathrm{~s}, 1 \mathrm{H}), 2.31(\mathrm{~s}, 3 \mathrm{H}) ;{ }^{13} \mathrm{C}-\mathrm{NMR}\left(100 \mathrm{MHz}, \mathrm{CDCl}_{3}, \mathrm{ppm}\right) \delta 142.7,136.4$, $134.6(q, J=33 \mathrm{~Hz}), 132.9,130.1,127.8,126.1(\mathrm{q}, J=3.7 \mathrm{~Hz}), 123.2(\mathrm{q}, J=271.1 \mathrm{~Hz}), 123.0,20.8$; MS (EI) $m / z(\%) 315,145,106(100), 77$.

N-p-Tolymethanesulfonamide (3u) [55]: Off-white solid, 71\% yield (26.3 mg), ${ }^{1} \mathrm{H}-\mathrm{NMR}\left(400 \mathrm{MHz} \mathrm{CDCl}_{3}\right.$, ppm) $\delta 7.16(\mathrm{~d}, J=7.2 \mathrm{~Hz}, 4 \mathrm{H}), 6.46(\mathrm{~s}, 1 \mathrm{H}), 3.00(\mathrm{~s}, 3 \mathrm{H}), 2.36(\mathrm{~s}, 3 \mathrm{H}) ;{ }^{13} \mathrm{C}-\mathrm{NMR}\left(100 \mathrm{MHz}, \mathrm{CDCl}_{3}, \mathrm{ppm}\right)$ $\delta 135.6,134.1,130.2,121.7,39.0,20.8$; MS (EI) $m / z$ (\%) 185, 106 (100), 79.

\section{Conclusions}

In summary, we have developed a simple synthetic method for the preparation of $N$-arylsulfonamides via copper-catalyzed redox coupling of sodium sulfinates and nitroarenes. Aromatic sulfonamides were formed in moderate to good yields in the absence of external oxidant or reductant. Active functional groups such as ester, cyano, and halogens were well tolerated under the optimized conditions. This method affords an alternative route for the synthesis of $N$-aryl sulfonamides under mild conditions. Moreover, this protocol would inspire other cases of nitroarene reduction-based synthesis of complex compounds. The detailed mechanism and the synthetic applications of this reaction are currently under investigation.

Supplementary Materials: The supplementary materials are available online.

Author Contributions: Conceptualization, S.L. and J.Z.; methodology, R.C.; writing-original draft preparation, S.L.; writing-review and editing, S.L.

Funding: We gratefully thank the Normal Project Foundation of Hunan Provincial Education Department (18C0845) and the Key Project Foundation of Hunan Provincial Education Department (18A396).

Conflicts of Interest: The authors declare no conflict of interest.

\section{References}

1. Kerr, J.M.; Suckling, C.J.; Bamfield, P. Selective Hydrogenation by A Novel Palladium(II) Complex. Tetrahedron Lett. 1988, 29, 5545-5548. [CrossRef]

2. Zhao, F.Y.; Zhang, R.; Chatterjee, M.; Ikushima, Y.; Arai, M. Hydrogenation of Nitrobenzene with Supported Transition Metal Catalysts in Supercritical Carbon Dioxide. Adv. Synth. Catal. 2004, 346, 661-668. [CrossRef]

3. Corma, A.; Serna, P.; Concepcion, P.; Calvino, J.J. Transforming Nonselective into Chemoselective Metal Catalysts for the Hydrogenation of Substituted Nitroaromatics. J. Am. Chem. Soc. 2008, 130, 8748-8753. [CrossRef]

4. Cantillo, D.; Baghbanzadeh, M.; Kappe, C.O. In Situ Generated Iron Oxide Nanocrystals as Efficient and Selective Catalysts for the Reduction of Nitroarenes using a Continuous Flow Method. Angew. Chem. Int. Ed. 2012, 51, 10190-10193. [CrossRef]

5. Jagadeesh, R.V.; Surkus, A.E.; Junge, H.; Pohl, M.M.; Radnik, J.; Rabeah, J.; Huan, H.; Schünemann, V.; Brückner, A.; Beller, M. Nanoscale $\mathrm{Fe}_{2} \mathrm{O}_{3}$-Based Catalysts for Selective Hydrogenation of Nitroarenes to Anilines. Science 2013, 342, 1073-1076. [CrossRef] [PubMed]

6. Zhao, Z.K.; Yang, H.L.; Li, Y.; Guo, X.W. Cobalt-Modified Molybdenum Carbide as an Efficient Catalyst for Chemoselective Reduction of Aromatic Nitro Compounds. Green Chem. 2014, 16, 1274-1281. [CrossRef]

7. Ono, N. The Nitro Group in Organic Synthesis; Wiley-VCH: New York, NY, USA, 2001.

8. Lawrence, S. Amines: Synthesis, Properties and Applications; Cambridge University Press: Cambridge, UK, 2004.

9. Arnold, H.; Döbert, F.; Gaube, J. In Handbook of Heterogeneous Catalysis; Wiley-Interscience: New York, NY, USA, 2008; pp. 3266-3284. 
10. Srivastava, R.S.; Nicholas, K.M. Iron-Catalyzed Allylic Amination by Nitroorganics. Chem. Comm. 1998, 24, 2705-2706. [CrossRef]

11. Chen, J.Z.; Ling, G.; Lu, S.W. Synthesis of N-Phenyl-N'-pyrimidylurea Derivatives by Selenium- or Selenium Dioxide-Catalyzed Reductive Carbonylation of Nitroaromatics. Eur. J. Org. Chem. 2003, 17, 3446-3452. [CrossRef]

12. Li, M.; Hu, L.Q.; Cao, X.H.; Hong, Y.; Lu, J.M.; Gu, H.W. Direct Hydrogenation of Nitroaromatics and One-Pot Amidation with Carboxylic Acids over Platinum Nanowires. Chem. Eur. J. 2011, 17, 2763-2768. [CrossRef] [PubMed]

13. Gui, J.; Pan, C.; Jing, Y.; Qin, T.; Lo, J.; Lee, B.; Spergel, S.; Mertzman, M.; Pitts, W.; Cruz, T.; et al. Practical Olefin Hydroamination with Nitroarenes. Science 2015, 348, 886-891. [CrossRef]

14. Oakdale, J.; Solano, D.; Fettinger, J.; Haddadin, M.; Kurth, M.J. An Oxazolo[3,2-b]indazole Route to 1H-Indazolones. Org. Lett. 2009, 11, 2760-2763. [CrossRef]

15. Cui, X.J.; Zhang, Y.; Shi, F.; Deng, Y.Q. Ruthenium-Catalyzed Nitro and Nitrile Compounds Coupling with Alcohols: Alternative Route for N-Substituted Amine Synthesis. Chem. Eur. J. 2011, 17, 2587-2591. [CrossRef]

16. Zanardi, A.; Mata, J.A.; Peris, E. One-Pot Preparation of Imines from Nitroarenes by a Tandem Process with an Ir-Pd Heterodimetallic Catalyst. Chem. Eur. J. 2010, 16, 10502-10506. [CrossRef]

17. Tang, C.H.; He, L.; Liu, Y.M.; Cao, Y.; He, H.Y.; Fan, K.N. Direct One-Pot Reductive N-Alkylation of Nitroarenes by using Alcohols with Supported Gold Catalysts. Chem. Eur. J. 2011, 17, 7172-7177. [CrossRef]

18. Nguyen, T.B.; Ermolenko, L.; Al-Mourabit, A. Iron Sulfide Catalyzed Redox/Condensation Cascade Reaction between 2-Amino/Hydroxy Nitrobenzenes and Activated Methyl Groups: A Straightforward Atom Economical Approach to 2-Hetaryl-benzimidazoles and -benzoxazoles. J. Am. Chem. Soc. 2013, 135, 118-121. [CrossRef]

19. Cui, X.J.; Deng, Y.Q.; Shi, F. Reductive N-Alkylation of Nitro Compounds to N-Alkyl and N,N-Dialkyl Amines with Glycerol as the Hydrogen Source. ACS Catal. 2013, 3, 808-811. [CrossRef]

20. Feng, C.; Liu, Y.; Peng, S.M.; Shuai, Q.; Deng, G.J.; Li, C.J. Ruthenium-Catalyzed Tertiary Amine Formation from Nitroarenes and Alcohols. Org. Lett. 2010, 12, 4888-4891. [CrossRef]

21. Liu, Y.; Chen, W.; Feng, C.; Deng, G.J. Ruthenium-Catalyzed One-Pot Aromatic Secondary Amine Formation from Nitroarenes and Alcohols. Chem. Asian J. 2011, 6, 1142-1146. [CrossRef]

22. Cano, R.; Ramón, D.J.; Yus, M. Impregnated Ruthenium on Magnetite as a Recyclable Catalyst for the $\mathrm{N}$-Alkylation of Amines, Sulfonamides, Sulfinamides, and Nitroarenes Using Alcohols as Electrophiles by a Hydrogen Autotransfer Process. J. Org. Chem. 2011, 76, 5547-5557. [CrossRef]

23. Xiao, F.H.; Liu, Y.; Tang, C.L.; Deng, G.J. Peroxide-Mediated Transition-Metal-Free Direct Amidation of Alcohols with Nitroarenes. Org. Lett. 2012, 14, 984-987. [CrossRef]

24. Wu, M.Y.; Hu, X.; Liu, J.; Liao, Y.F.; Deng, G.J. Iron-Catalyzed 2-Arylbenzoxazole Formation from o-Nitrophenols and Benzylic Alcohols. Org. Lett. 2012, 14, 2722-2725. [CrossRef]

25. Wang, H.M.; Cao, X.X.; Xiao, F.H.; Liu, S.W.; Deng, G.J. Iron-Catalyzed One-Pot 2,3-Diarylquinazolinone Formation from 2-Nitrobenzamides and Alcohols. Org. Lett. 2013, 15, 4900-4903. [CrossRef]

26. Wang, H.M.; Chen, H.; Chen, Y.; Deng, G.J. Palladium-Catalyzed One Pot 2-Arylquinazoline Formation via Hydrogen-Transfer Strategy. Org. Biomol. Chem. 2014, 12, 7792-7799. [CrossRef]

27. Nguyen, T.B.; Ermolenko, L.; Al-Mourabit, A. Nitro-Methyl Redox Coupling: Efficient Approach to 2-Hetarylbenzothiazoles from 2-Halonitroarene, Methylhetarene, and Elemental Sulfur. Org. Lett. 2013, 15, 4218-4221. [CrossRef]

28. Nguyen, T.B.; Retailleau, P.; Al-Mourabit, A. A Simple and Straightforward Approach to Quinoxalines by Iron/Sulfur-Catalyzed Redox Condensation of $o$-Nitroanilines and Phenethylamines. Org. Lett. 2013, 15, 5238-5241. [CrossRef]

29. Nguyen, T.B.; Bescont, J.L.; Ermolenko, L.; Al-Mourabit, A. Cobalt- and Iron-Catalyzed Redox Condensation of $o$-Substituted Nitrobenzenes with Alkylamines: A Step- and Redox-Economical Synthesis of Diazaheterocycles. Org. Lett. 2013, 15, 6218-6221. [CrossRef]

30. Nguyen, T.B.; Pasturaud, K.; Ermolenko, L.; Al-Mourabit, A. Concise Access to 2-Aroylbenzothiazoles by Redox Condensation Reaction between o-Halonitrobenzenes, Acetophenones, and Elemental Sulfur. Org. Lett. 2015, 17, 2562-2565. [CrossRef] 
31. Xing, Q.Y.; Ma, Y.F.; Xie, H.; Xiao, F.H.; Zhang, F.; Deng, G.J. Iron-Promoted Three-Component 2-Substituted Benzothiazole Formation via Nitroarene ortho C-H Sulfuration with Elemental Sulfur. J. Org. Chem. 2019, 84, 1238-1246. [CrossRef]

32. Ganguly, A.K.; Alluri, S.S.; Caroccia, D.; Biswas, D.; Wang, C.H.; Kang, E.; Zhang, Y.; McPhail, A.T.; Carroll, S.S.; Burlein, C.; et al. Design, Synthesis, and X-ray Crystallographic Analysis of a Novel Class of HIV-1 Protease Inhibitors. J. Med. Chem. 2011, 54, 7176-7183. [CrossRef]

33. Gilbert, A.M.; Caltabiano, S.; Koehn, F.E.; Chen, Z.-J.; Francisco, G.D.; Ellingboe, J.W.; Kharode, Y.; Mangine, A.; Francis, R.; TrailSmith, M.; et al. Pyrazolopyrimidine-2,4-dione Sulfonamides: Novel and Selective Calcitonin Inducers. J. Med. Chem. 2002, 45, 2342-2345. [CrossRef]

34. Masereel, B.; Rolin, S.; Abbate, F.; Scozzafava, A.; Supuran, C.T. Carbonic Anhydrase Inhibitors: Anticonvulsant Sulfonamides Incorporating Valproyl and Other Lipophilic Moieties. J. Med. Chem. 2002, 45, 312-320. [CrossRef]

35. Drew, J. Drug Discovery: A Historical Perspective. Science 2000, 287, 1960-1964. [CrossRef]

36. Wilden, J.; Geldeard, L.; Lee, C.; Judd, D.; Caddick, S. Trichlorophenol (TCP) Sulfonate Esters: A Selective Alternative to Pentafluorophenol (PFP) Esters and Sulfonyl Chlorides for The Preparation of Sulfonamides. Chem. Commun. 2007, 10, 1074-1076. [CrossRef]

37. Bahrami, K.; Khodaei, M.; Soheilizad, M. Direct Conversion of Thiols to Sulfonyl Chlorides and Sulfonamides. J. Org. Chem. 2009, 74, 9287-9291. [CrossRef]

38. Zhu, M.; Wei, W.; Yang, D.; Cui, H.; Wang, L.; Meng, G.; Wang, H. Metal-Free $\mathrm{I}_{2} \mathrm{O}_{5}$-Mediated Direct Construction of Sulfonamides from Thiols and Amines. Org. Biomol. Chem. 2017, 15, 4789-4793. [CrossRef]

39. Sheykhan, M.; Khani, S.; Abbasnia, M.; Shaabanzadeh, S.; Joafshan, M. An Approach to C-N Activation: Coupling of Arenesulfonyl Hydrazides and Arenesulfonyl Chlorides with Tert-Amines via A Metal-, Oxidantand Halogen-free Anodic Oxidation. Green Chem. 2017, 19, 5940-5948. [CrossRef]

40. DeBergh, J.R.; Niljianskul, N.; Buchwald, S.L. Synthesis of Aryl Sulfonamides via Palladium-Catalyzed Chlorosulfonylation of Arylboronic Acids. J. Am. Chem. Soc. 2013, 135, 10638-10641. [CrossRef]

41. Tang, X.; Huang, L.; Qi, C.; Wu, X.; Wu, W.; Jiang, H. Copper-Catalyzed Sulfonamides Formation from Sodium Sulfinates and Amines. Chem. Commun. 2013, 49, 6102-6104. [CrossRef]

42. Buathongjan, C.; Beukeaw, D.; Yotphan, S. Iodine-Catalyzed Oxidative Amination of Sodium Sulfinates: A Convenient Approach to the Synthesis of Sulfonamides under Mild Conditions. Eur. J. Org. Chem. 2015, 7, 1575-1582. [CrossRef]

43. Pan, X.; Gao, J.; Liu, J.; Lai, J.; Jiang, H.; Yuan, G. Synthesis of Sulfonamides via I2-Mediated Reaction of Sodium Sulfinates with Amines in An Aqueous Medium at Room Temperature. Green. Chem. 2015, 17, 1400-1403. [CrossRef]

44. Yang, K.; Ke, M.; Lin, Y.; Song, Q. Sulfonamide Formation from Sodium Sulfinates and Amines or Ammonia under Metal-free Conditions at Ambient Temperature. Green Chem. 2015, 17, 1395-1399. [CrossRef]

45. Sarkar, D.; Ghosh, M.K.; Rout, N. PTAB Mediated Open Air Synthesis of Sulfonamides, Thiosulfonates and Symmetrical Disulfanes. Tetrahedron Lett. 2018, 59, 2360-2364. [CrossRef]

46. Baffoe, J.; Hoe, M.; Touré, B. Copper-Mediated N-Heteroarylation of Primary Sulfonamides: Synthesis of Mono-N-heteroaryl Sulfonamides. Org. Lett. 2010, 12, 1532-1535. [CrossRef]

47. Burton, G.; Cao, P.; Li, G.; Rivero, R. Palladium-Catalyzed Intermolecular Coupling of Aryl Chlorides and Sulfonamides under Microwave Irradiation. Org. Lett. 2003, 5, 4373-4376. [CrossRef]

48. Rosen, B.R.; Ruble, J.C.; Beauchamp, T.J.; Navarro, A. Mild Pd-Catalyzed N-Arylation of Methanesulfonamide and Related Nucleophiles: Avoiding Potentially Genotoxic Reagents and Byproducts. Org. Lett. 2011, 13, 2564-2567. [CrossRef]

49. Nasrollahzadeh, M.; Ehsani, A.; Maham, M. Copper-Catalyzed N-Arylation of Sulfonamides with Boronic Acids in Water under Ligand-Free and Aerobic Conditions. Synlett 2014, 25, 505-508. [CrossRef]

50. Nasrollahzadeha, M.; Rostami-Vartooni, A.; Ehsani, A.; Moghadam, M. Fabrication, Characterization and Application of Nanopolymer Supported Copper (II) Complex as an Effective and Reusable Catalyst for the C-N Bond Cross-Coupling Reaction of Sulfonamides with Arylboronic Acids in Water under Aerobic Conditions. J. Mol. Catal. A: Chem. 2014, 387, 123-129. [CrossRef]

51. Cao, X.; Bai, Y.; Xie, Y.; Deng, G.J. Palladium-Catalyzed Arylation of Aryl Sulfonamides with Cyclohexanones. J. Mol. Catal. A: Chem. 2014, 383, 94-100. [CrossRef] 
52. Qu, P.; Sun, C.; Ma, J.; Li, F. The N-Alkylation of Sulfonamides with Alcohols in Water Catalyzed by the Water-Soluble Iridium Complex $\left\{\mathrm{Cp}^{*} \operatorname{Ir}\left[6,6(\mathrm{OH})_{2} \mathrm{bpy}\right]\left(\mathrm{H}_{2} \mathrm{O}\right)\right\}[\mathrm{OTf}]_{2}$. Adv. Synth. Catal. 2014, 356, 447-459. [CrossRef]

53. Ramachandran, R.; Prakash, G.; Selvamurugan, S.; Viswanathamurthi, P.; Malecki, J.G.; Ramkumar, V. Efficient and Aersatile Catalysis of $N$-alkylation of Heterocyclic Amines with Alcohols and One-Pot Synthesis of 2-Aryl Substituted Benzazoles with Newly Designed Ruthenium(II) Complexes of PNS Thiosemicarbazones. Dalton Trans. 2014, 43, 7889-7902. [CrossRef]

54. Li, Q.Q.; Xiao, Z.F.; Yao, C.Z.; Zheng, H.X.; Kang, Y.B. Direct Alkylation of Amines with Alcohols Catalyzed by Base. Org. Lett. 2015, 17, 5328-5331. [CrossRef]

55. Shekhar, S.; Dunn, T.B.; Kotechi, B.; Montavon, D.; Cullen, S.C. A General Method for Palladium-Catalyzed Reactions of Primary Sulfonamides with Aryl Nonaflates. J. Org. Chem. 2011, 76, 4552-4563. [CrossRef]

56. Xiao, B.; Gong, T.J.; Xu, J.; Liu, Z.J.; Liu, L. Palladium-Catalyzed Intermolecular Directed C-H Amidation of Aromatic Ketones. J. Am. Chem. Soc. 2011, 133, 1466-1474. [CrossRef]

57. Zhao, H.Q.; Shang, Y.P.; Su, W.P. Rhodium(III)-Catalyzed Intermolecular N-Chelator-Directed Aromatic C-H Amidation with Amides. Org. Lett. 2013, 15, 5106-5109. [CrossRef]

58. Zhang, W.; Xie, J.; Rao, B.; Luo, M. Iron-Catalyzed N-Arylsulfonamide Formation through Directly Using Nitroarenes as Nitrogen Sources. J. Org. Chem. 2015, 80, 3504-3511. [CrossRef]

59. Li, X.X.; Chen, F.; Lu, G.P. Fe-Based Metal-Organic Frameworks for The Synthesis of N-Arylsulfonamides via The Reactions of Sodium Arylsulfinates or Arylsulfonyl Chlorides with Nitroarenes in Water. Tetrahedron Lett. 2018, 59, 4226-4230. [CrossRef]

60. Nadim, E.; Karamé, I.; Andrioletti, B. Straightforward and Sustainable Synthesis of Sulfonamides in Water under Mild Conditions. Eur. J. Org. Chem. 2018, 5016-5022.

61. Jiang, J.; Zeng, S.; Chen, D.; Cheng, C.; Deng, W.; Xiang, J. Synthesis of N-Arylsulfonamides via Fe-Promoted Reaction of Sulfonyl Halides with Nitroarenes in an Aqueous Medium. Org. Biomol. Chem. 2018, 16, 5016-5020. [CrossRef]

62. Zhao, F.; Li, B.; Huang, H.W.; Deng, G.J. Palladium-Catalyzed N-Arylsulfonamide Formation from Arylsulfonyl Hydrazides and Nitroarenes. RSC Adv. 2016, 6, 13010-13013. [CrossRef]

63. Yang, B.; Lian, C.; Yue, G.L.; Liu, D.Y.; Wei, L.Y.; Ding, Y.; Zheng, X.C.; Lu, K.; Qiu, D.; Zhao, X. Synthesis of $\mathrm{N}$-Arylsulfonamides Through A Pd-Catalyzed Reduction Coupling Reaction of Nitroarenes with Sodium Arylsulfinates. Org. Biomol. Chem. 2018, 16, 8150-8154. [CrossRef]

64. Deruer, E.; Coulibali, S.; Boukercha, S.; Canesi, S. Carbon-Phosphorus Bond Formation on Anilines Mediated by a Hypervalent Iodine Reagent. J. Org. Chem. 2017, 82, 11884-11890. [CrossRef]

65. Downer, N.K.; Jackson, Y.A. Synthesis of Benzothiazoles via Ipso Substitution of Orthomethoxythiobenzamides. Org. Biomol. Chem. 2004, 2, 3039-3043. [CrossRef]

66. Natarajan, A.; Guo, Y.H.; Harbinski, F.; Fan, Y.H.; Chen, H.; Luus, L.; Diercks, J.; Aktas, H.; Chorev, M.; Halperin, J.A. Novel Arylsulfoanilide-Oxindole Hybrid as an Anticancer Agent That Inhibits Translation Initiation. J. Med. Chem. 2004, 47, 4979-4982. [CrossRef]

67. Aissaoui, H.; Koberstein, R.; Zumbrunn, C.; Gatfield, J.; Brisbare-Roch, C.; Jenck, F.; Treiber, A.; Boss, C. $\mathrm{N}$-Glycine-sulfonamides as Potent Dual Orexin 1/Orexin 2 Receptor Antagonists. Bioorg. Med. Chem. Lett. 2008, 18, 5729-5733. [CrossRef]

68. Sadaf, A.; Priyanka, C.; Popuri, S.; Shahulhameed, S.; Jeyakumar, K. tert-Butyl Nitrite Mediated Nitrogen Transfer Reactions: Synthesis of Benzotriazoles and Azides at Room Temperature. Org. Biomol. Chem. 2018, $16,8280-8285$.

Sample Availability: Not available. 